\title{
Influence of Ohmic contacts on the amplitude of Shubnikov-de Haas oscillations
}

\author{
K von Klitzing, J Nieder, R J Haug, G Müller, S Koch, D Weiss \\ and K Ploog
}

Max-Planck-Institut für Festkörperforschung, Heisenbergstrasse 1, D-7000 Stuttgart 80, Federal Republic of Germany

Received 18 June 1991, accepted for publication 7 August 1991

Abstract. The longitudinal magnetoresistance of a two-dimensional electron gas is shown to be strongly dependent on the presence of ohmic contacts between the voltage probes. By connecting or disconnecting contacts with additional gates across the potential probes it is possible to drastically change the amplitude of Shubnikov-de Haas oscillations.

In an early work of Palaanen et al [1], an anomalous suppression of the Shubnikov-de Haas (SDH) maxima was observed in a standard $\mathrm{AlGaAs} / \mathrm{GaAs}$ heterostructure, but the interpretation remained obscure. In the last few years, the Landauer-Büttiker (LB) formalism [2-4] has established a description of magneto-quantum transport phenomena in terms of the edge channel picture [5]. Within this picture, ohmic contacts serve as energy dissipating, distribution equilibrating and phase randomizing electron reservoirs. In real samples nonideal ohmic contacts may be present due to an imperfect alloying [6,7]. Such a disordered contact reflects (at least partly) incoming edge channels. This behaviour can be simulated by artificially suppressing the transmission of edge channels into an ohmic contact $\%$. One technique for the realization of this is to selectively fabricate a split gate [8] or a Schottky gate [9] across the leads to the contact to detect and populate only the outer edge channels, another is to fabricate a Corbino contact [10] or a pseudo Corbino contact [11] to detect and populate only the inner edge channels. Several interesting magnetotransport experiments $[6,7,8-14]$ convincingly confirm the edge channel picture and particularly the possibility of selectively populating and detecting only a fraction of edge states, which gives rise to the observa-

$\dagger$ The terminology used in this paper is as follows: The lowest (energy) Landau level provides the lowest (energy) edge state equivalent to the outermost (located) edge channel. The highest occupied Landau level provides the highest edge state equivalent to the innermost edge channel (compare with figure 2). The Landau level index is $n$, where an arrow shows the direction of the resolved spin-split level (for example: $n=0 \dagger$ ). There are $N$ landau levels at or below the Fermi energy $E_{\mathrm{F}}$. If $N$ is an odd (even) number, a spin-up (spindown) level is the uppermost one. tion uI novel phenomena, e.g., in high mobility samples a non-equilibrium electron distribution between different edge states over macroscopic distances $[6,7,9,13,14]$.

All the experimental works cited above investigate the situation in the quantum Hall rcgime. Between the quantum Hall plateaux, backscattering within the uppermost edge state causes dissipation. Whereas the uppermost state extends into the interior of the sample, all other $N-1$ states are supposed to be still localized at the sample edges. The decoupling of the uppermost edge state is reminescent of the dissipative regime. This decoupling leads to a length independent resistance contribution of the lower edge states [15]. Van Wees et al [16] have observed for the $N=3$ situation a suppression of the $n=1 \uparrow \mathrm{SDH}$ peak in a three-terminal measurement using a disordered contact simulated by a split gate across the lead to selectively detect the two lower states only. This again implies a perfect decoupling of the uppermost edge state and stresses the exceptional role of the $N=3$ situation.

In this paper we study the dissipative regime in magneto-quantum transport measurements, i.e. the maxima of the SDH oscillations. We are able to simulate a change of the energy dissipation on the same sample by means of connecting ohmic contacts to the 2DEG between the voltage probes on a sample without any barriers across the Hall bar. We compare the influence of different numbers of contacts and discuss temperature and current dependences.

For the magnetotransport measurements a standard $\mathrm{Al}_{x} \mathrm{Ga}_{1-x} \mathrm{As} / \mathrm{GaAs}$ heterostructure $(x=0.33$ ) is used. The sheet electron density and the mobility at liquid Helium temperatures are $n_{s}=2.9 \times 10^{11} \mathrm{~cm}^{-2}$ and $\mu=650000 \mathrm{~cm}^{2} \mathrm{~V}^{-1} \mathrm{~s}^{-1}$ respectively, which yields a 


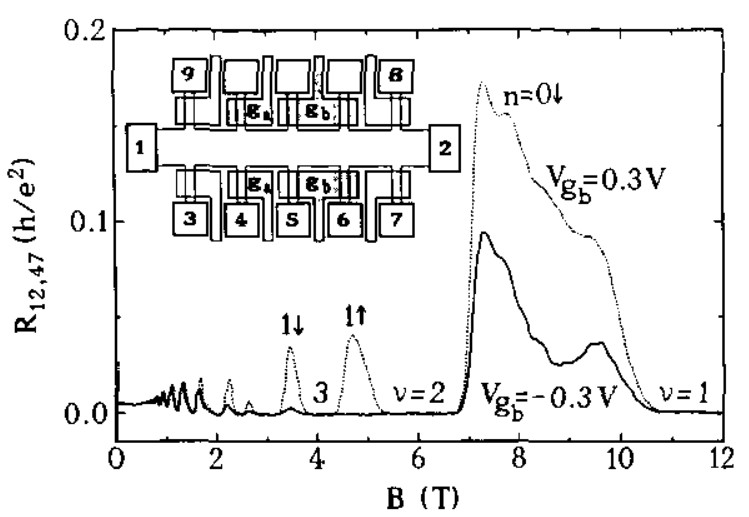

Figure 1. Longitudinal magnetoresistance $R_{12,47}=U_{47} / I_{12}$ of an AlGaAs/GaAs heterostructure. The geometry of the device is sketched in the inset.

Drude mean free path of $l=5.9 \mu \mathrm{m}$. By means of optical lithography and wet etching a standard Hall bar is fabricated (see inset of figure 1). The width of the Hall bar is $W=40 \mu \mathrm{m}$, the distance between adjacent voltage contacts is $l=60 \mu \mathrm{m}$. Cr/Au gates (width $25 \mu \mathrm{m}$ ) are evaporated across the leads of the voltage contacts. The sample geometry is shown in the inset of figure 1. Standard lock-in techniques at a frequency of $13.5 \mathrm{~Hz}$ are used. The magnetic field is always applied perpendicular to the 2DEG. The measurements have been performed in a $\mathrm{He}^{3} / \mathrm{He}^{4}$ dilution refrigerator. The bath temperature for all measurements is $T=35 \mathrm{mK}$, the actual electron temperature is probably somewhat higher.

Figure 1 shows the four-terminal magnetoresistance $R_{i j, k l}(B)=U_{k l} / I_{i j}=R_{12,47}$ with $I_{i j}$ the current from contact $i$ to $j\left(I_{12}=10 \mathrm{nA}\right)$ and $U_{k l}$ the voltage between the contacts $k$ and $l . R_{12,47}$ is plotted as a dotted line for $V_{\mathrm{g}_{\mathrm{b}}}=0.3 \mathrm{~V}$ at gate $\mathrm{g}_{\mathrm{b}}$, corresponding to connected contacts, whereas $R_{12,47}$ is plotted as a full line for $V_{g_{b}}=-0.3 \mathrm{~V}$ corresponding to the disconnection of all four contacts between the voltage probes 4 and 7 . All other gates across the leads to the voltage contacts are at $V_{\mathrm{gb}}=0 \mathrm{~V}$. The gate voltages are applied with respect to current contact 1 . In contrast to the situation $V_{\mathrm{g}_{\mathrm{b}}}=+0.3 \mathrm{~V}$, where all contacts between the voltage probes 4 and 7 are electrically connected to the Hall bar, the Shubnikov-de Haas oscillations are strongly suppressed for the situation $V_{\mathrm{go}}=-0.3 \mathrm{~V}$, where electrons do not recognize any reservoir along the current path between the voltage probes 4 and 7 . We want to emphasize that neither the current path $1-2$, nor the voltage probes 4 and 7 are directly affected by tuning gate $g_{\mathrm{b}}$. For the four-terminal resistance $R_{12,37}$ it is now possible to independently tune the two gates $g_{a}$ and $g_{b}$ (see inset of figure 1) between the voltage probes. The SDH amplitude $R_{12,37}$ for $B>1 \mathrm{~T}$ increases with increasing number of connected contacts. The $n=1 \uparrow$ peak vanishes again if both gates $g_{a}$ and $g_{b}$ are negatively biased to disconnect all six contacts between the voltage probes.

The starting point for the interpretation is the as-

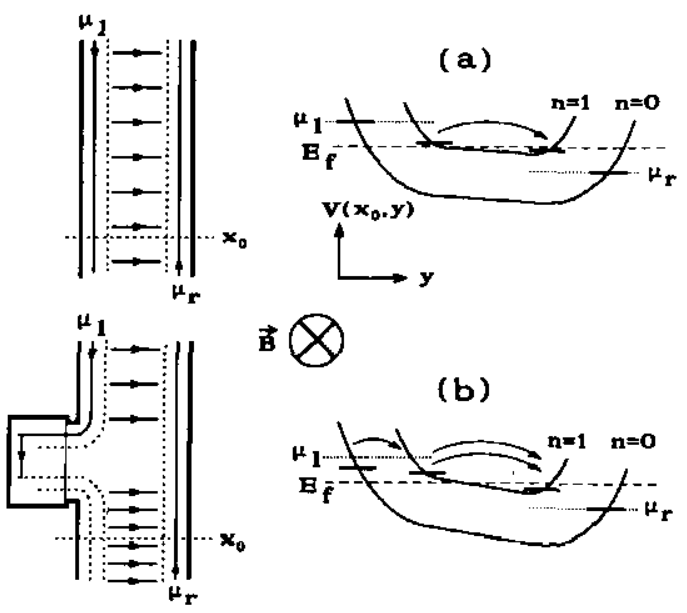

Figure 2. Representation of the simulation of enhanced interedge channel scattering by means of an ohmic contact. On the left-hand side the spatial current distribution along the Hall bar is sketched. On the right-hand side the potential cross section across the Hall bar at $x_{0}$ for two Landau levels is drawn. (a) Situation without an ohmic contact: The upper $n=1$ state coincides with the Fermi energy $E_{F}$ and provides electrons scattering from left to right without any contribution of the lower $n=0$ edge state, which remains at the electrochemical potential $\mu_{\mu}$. (b) Situation with an ohmic contact: The different electrochemical potentials of the left channels entering the contact become equilibrated. The contact acts as an electron reservoir, which transfers electrons from the outer channel to the inner one and thus increases the rate of electrons moving from left to right.

sumption that the uppermost edge state is decoupled from all the lower edge states. This is due to a reduced interedge channel scattering rate when the innermost state loses its edge channel character as it extends into the interior of the sample. The influence of connecting or disconnecting ohmic contacts to the 2DEG is sketched in figure 2. Consider a part of a Hall bar in a perpendicular magnetic field drawn on the left. The full lines represent the ideal transmitting outermost edge channels, which are equipotential lines, dotted lines represent extended states. Corresponding to that, the potential distribution $V\left(x_{0}, y\right)$ across the Hall bar at $x_{0}$ is drawn on the right. For sake of simplicity only two states are considered, which does not affect the principle of the mechanism. $\mu_{l}$ and $\mu_{r}$ are the electrochemical potentials of the outermost edge channels. For a noninteger filling factor $\nu$, i.e. in the dissipative regime, the Fermi energy coincides with the uppermost Landau level, which enables intra Landau level scattering from left to right. If no interedge channel scattering occurs (see figure 2(a)), only the uppermost state contributes to the backscattering process. If an ideal ohmic contact is connected to the sample (see figure $2(b)$ ), it acts as a potential equilibrating electron reservoir, i.e. additional electrons from the completely filled outermost channel are transferred into the extended state, which simulates an enhancement of the intra Landau level scattering 


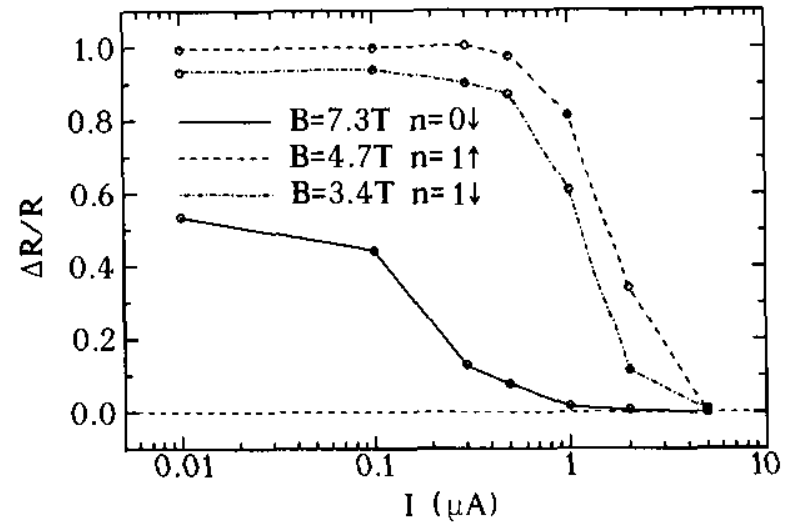

Figure 3. Current dependence of the normalized resistance difference $\Delta R_{12,47} / R_{12,47}$ between the resistances for two different gate voltages $V_{g}=+0.3 \mathrm{~V}$ and $-0.3 \mathrm{~V}$ of figure 1 for three Shubnikov-de Haas maxima at $n=0 \downarrow, n=1 \uparrow$ and $n=1 \downarrow$. The lines drawn between the data points are guides for the eye.

rate and causes an increase of the SDH maximum.

Surprisingly the SDH peak at $4.7 \mathrm{~T}$, which corresponds to the situation of the fully occupied $n=0 \uparrow$ and $n=0 \downarrow$ levels and the half-filled $n=1 \uparrow$ level, van- ishes. This strong effect can be explained by a perfect decoupling of the inner edge channels and a reflection of the uppermost channel at the gates across the voltage leads.

Note that to observe this effect a non-equilibrium distribution between the edge channels has to remain at least over the distance between the voltage probes, which is $180 \mu \mathrm{m}$ for $R_{12,47}$ and $240 \mu \mathrm{m}$ for $R_{12,37}$. A detectable $R_{12,47}$ difference between the two situations with connected or disconnected ohmic contacts can be observed starting at $B=1 \mathrm{~T}(\nu=12)$. It is surprising that even for a large number of edge channels and low magnetic fields, where the distance between adjacent edge states is small, a non-equilibrium population between the edge states is establishcd. The maximum suppression of $R_{x x}$ is observed at $B=4.7 \mathrm{~T}$ for the $n=1 \uparrow$ state between filling factor $\nu=2$ and $\nu=3$. Another observation is the unexpected high $R_{12,47}$ difference, which is found for the $n=0 \downarrow$ state between filling factor $\nu=1$ and $\nu=2$ and for the $n=1 \downarrow$ state between $\nu=3$ and $\nu=4$, where in both cases the decoupling of two spin states in the same Landau level occurs. The small spin splitting energy relative to the cyclotron energy $g \mu_{b} B \ll \hbar \omega_{c}$ leads to closely spaced spin resolved edge channels and the stronger wavefunc-
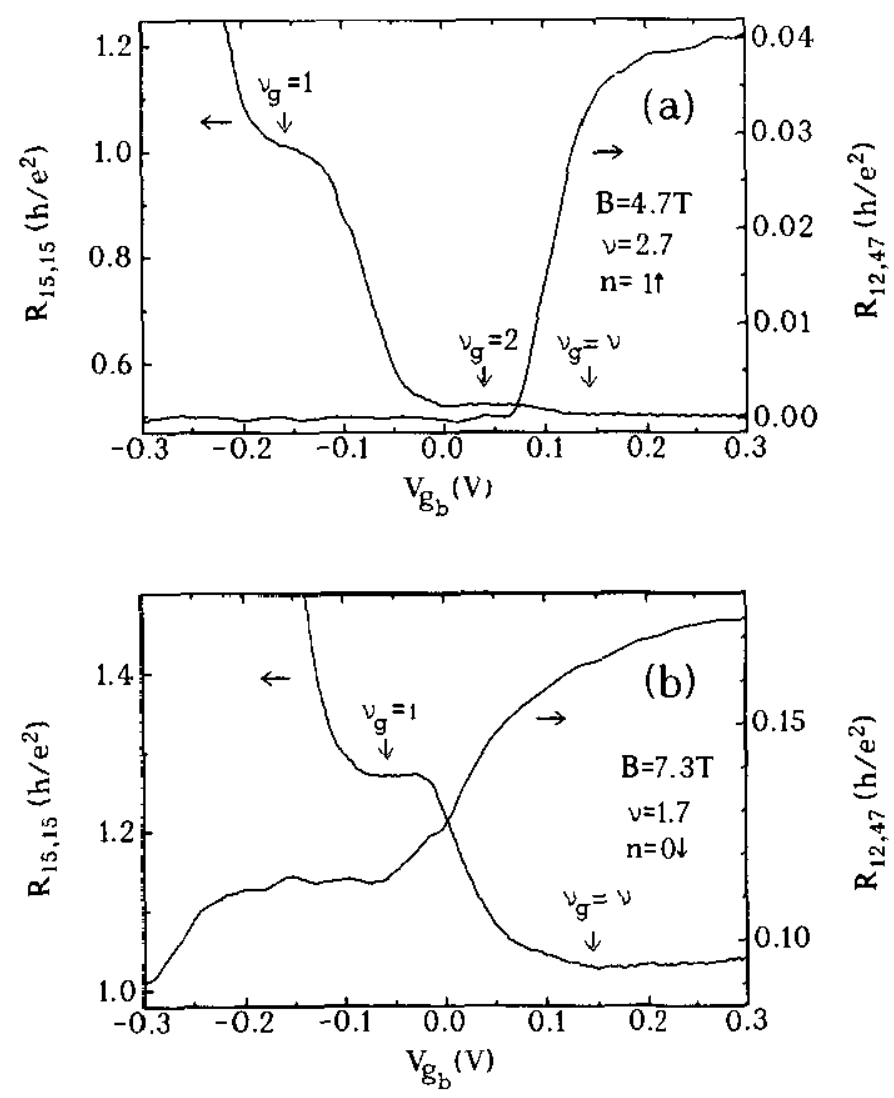

Figure 4. Comparison of the two-terminal resistance $A_{15,15}$ with the four-terminal resistance $R_{12,47}$ a a function of the gate voltage $V_{9 b}$ : (a) at the $n=1 \uparrow$ Shubnikov-de Haas maximum, (b) at the $n=0 \downarrow$ maximum. 
tion overlap should then lead to a more rapid dynamic equilibrium, i.e. to a small $R_{12,47}$ difference, as noted by Alphenaar et al [13]. As an extension of their results in the quantum Hall regime we propose that in the dissipative regime, where the innermost edge channel tends to move into the interior sample, in general the overlap of its wavefunction with the adjacent inner edge channel decreases and thus enhances the decoupling process, even between two spin states. In figure 3 the current dependence $[7,11]$ of the normalized resistance difference between connected and disconnected contacts $\Delta R / R=\left(R_{12,47}\left(V_{\mathrm{gb}}=+0.3 \mathrm{~V}\right)-R_{12,47}\left(V_{\mathrm{g} b}=\right.\right.$ $-0.3 \mathrm{~V})) / R_{12,47}\left(V_{\mathrm{g}_{\mathrm{b}}}=+0.3 \mathrm{~V}\right)$ of the SDH maxima is shown for three different magnetic fields. The values of $\Delta R / R$ for $I=0.01 \mu \mathrm{A}$ correspond to the values in figure 1. At low currents, $\Delta R / R$ is approximately independent of current. Note that for the $n=0 \downarrow$ case (full line), which represents the degree of partial decoupling between the two spin-splitted states of the $n=0$ Landau level, $\Delta R / R$ is much smaller than for the $n=1 \uparrow$ case (broken line), which represents a complete decoupling between the $n=0$ and $n=1$ Landau states. At certain critical currents $\Delta R / R$ drops to zero, i.e. all edge states become completely equilibrated. The highest critical current is observed for the $n=1 \uparrow$ situation which emphasizes that the decoupling process is most effective between the $n=0$ and $n=1$ Landau states ( $N=3$ situation). The relatively high critical current for the $n=1 \downarrow$ situation indicates that in this case the bottleneck for the interedge channel scattering is the decoupling between the $n=0 \downarrow$ and $n=1 \uparrow$ states and not (like in the $n=0 \downarrow$ situation) a decoupling between spin split states.

To demonstrate the contact induced simulation of enhanced interedge channel scattering in more detail, the four-terminal magnetoresistance $R_{12,47}$, plotted in figure 1 as a function of magnetic field, is now measured as a function of the gate voltage $V_{g_{b}}$. Figures $4(a)$ and $4(b)$ show the $n=1 \uparrow$ and the $n=0 \downarrow$ SDH maximum, respectively. In both figures, $R_{12,47}$ (right scale) is compared with the two-terminal resistance $R_{15,15}$ (left scale) between current contact 1 and contact 5 . In contrast to $R_{12,47}, R_{15,15}$ is directly influenced by $V_{\mathrm{g}}$ and is a measure of the number of channels transmitted into the reservoirs. At $V_{g_{b}}=-0.3 \mathrm{~V}$ for the $n=1 \uparrow$ situation (see figure $4(a)$ ),$R_{15,15}$ goes to infinity, i.e. no state can enter the equilibrating reservoir and $R_{12,47}$ is approximately zero. Even if $V_{g_{b}}$ is increased up to $0 \mathrm{~V}$ and $R_{15,15}$ shows that two states $\left(\nu_{\mathrm{g}}=2\right)$ are allowed to enter the reservoir, no increase of $R_{12,47}$ is observed, implying a complete equilibration of the two lower edge states. However, when the gate voltage exceeds $0.1 \mathrm{~V}$ and the filling factor beneath the gate reaches the bulk value $\left(\nu_{\mathrm{g}}=\nu\right)$, the uppermost state enters the reservoir to equilibrate with the others and causes a steep increase in $R_{12,47}$. A similar, although less steep increase is observed for the $n=0 \downarrow$ case shown in figure $4(b)$. Between $V_{g_{b}}=0 \mathrm{~V}$ and $0.1 \mathrm{~V}$ the $n=0 \downarrow$ state can enter the contacts to equilibrate with the $n=0 \uparrow$ state. The fact that $R_{12,47}$ is non-zero already before the $N=0 \downarrow$ spin state can enter the contacts for $-0.25 \mathrm{~V} \leqslant V_{\mathrm{go}_{\mathrm{b}}} \leqslant-0.05 \mathrm{~V}$, shows that the two spin states are only partially decoupled.

In conclusion it is possible to change the amplitude of Shubnikov-de Haas oscillations by tuning the transmission of edge states through unused contacts. This shows that connected contacts serve as equilibrating reservoirs for the decoupled states in the dissipative regime of the quantum Hall effect. We conclude that in higher mobility samples, where interedge channel scattering at impurities is effectively suppressed, Shubnikovde Haas oscillations are a manifestation of intrinsic nonequilibrium magnetotransport.

\section{Acknowledgments}

We are indebted to the group of $\mathrm{H} \mathrm{U}$ Habermeier, particularly I Skupin, S Tipmann, M Wurster and F Schartner for mask technology. We also thank A Gollhardt, $M$ Riek and $P$ Grambow for assisting the sample preparation and A D Wieck, E Diessel and O Viehweger for helpful discussions. We gratefully acknowledge financial support by the Bundesministerium für Forschung und Technologie.

\section{References}

[1] Paalanen M A, Tsui D C and Gossard A C 1982 Phys. Rev. B 255566

[2] Büttiker M 1988 Phys. Rev. B 389375

[3] Büttiker M 1986 Phys. Rev. Lett. 571761

[4] Büttiker M 1991. Nanostructured systems Semiconductor and Semimetals ed M Reed (New York: Academic) to be published

[5] Kane B E, Tsui D C and Weimann G 1987 Phys. Rev. Lett. 591353

[6] Komiyama S, Hirai H, Sasa S and Hiyamizu S 1989 Phys. Rev. B 4012566

[7] Komiyama S, Hirai H, Ohsawa M, Matruda H, Sasa S and Fujii T 1990 Proc. 20th Int. Conf. on the Physics of Semiconductors ed E M Anastassakis and J D Joannopoulos (Singapore: World Scientific) p 829

[8] van Wees B J, willems E M M, Harmans C J P M, Beenakker $\mathrm{C}$ W J, van Houten $\mathrm{H}$, Williamson $\mathrm{J} \mathrm{G}$, Foxon C T and Harris J J 1989 Phys. Rev. Lett. 62 1181

[9] Müller G, Weiss D, Koch S, von Klitzing K, Nickel H, Schlapp W and Lösch R 1990 Phys. Rev. B 427633

[10] Faist J, Guéret P and Meier H P 1991 Phys. Rev. B 43 9332

[11] Müller G, Diessel E, Weiss D, von Klitzing K, Ploog K, Nickel H, Schlapp W and Lösch R 1991 Proc. 9 th Conf. on Electronic Propenties of Two-Dimensional Systems to appear

[12] Haug R J, MacDonald A H, Streda P and von Klitzing K 1988 Phys. Rev. Lett. 612797

[13] Alphenaar B W, McEuen P L, Wheeler R G and Sacks R N 1990 Phys. Rev. Lett. 64677

[14] Alphenaar B W, McEuen P L, Wheeler R G and Sacks $\mathrm{R} N$ to be published

[15] Haug R J and von Klitzing K 1989 Europhys. Lett. 10 489

[16] van Wees B J, Willems E M M, Kouwenhoven L P, Harmans C J P M, Williamson J G, Foxon C T and Harris J J 1989 Phys. Rev. B 398066 